\title{
Multidimensional Inverse Scattering with Fixed-Energy Data
}

\author{
A.G. Ramm \\ Department of Mathematics, Kansas State University \\ Manhattan, KS 66506-2602, USA \\ ramm@math.ksu.edu
}

\section{Introduction}

In this lecture the author reviews his results on multidimensional inverse scattering. References to the works of other authors can be found in [20].

Five topics are briefly discussed:

- 1) property $C$ with constraints and new type of the uniqueness theorems for inverse scattering,

- 2) inversion of noisy discrete fixed-energy 3D scattering data and error estimates,

- 3) variational principles equivalent to inverse scattering problems,

- 4) low-frequency data inversion,

- 5) asymptotic inverse scattering theory for scattering by small inhomogeneities.

Detailed proofs of the results can be found in the cited references. In this paper the emphasis is on the ideas and formulation of the results.

The inverse problems we consider are: inverse potential, geophysical and obstacle scattering (IPS, IGS, IOS). We recall the statements of these problems. Let

$$
\begin{gathered}
{\left[\nabla^{2}+k^{2}-q(x)\right] u=0 \text { in } \mathbb{R}^{3}, \quad k=\text { const }>0} \\
u=\exp (i k \alpha \cdot x)+A\left(\alpha^{\prime}, \alpha, k\right) \frac{\exp (i k r)}{r}+o\left(\frac{1}{r}\right), \\
r:=|x| \rightarrow \infty, \alpha^{\prime}:=\frac{x}{r}, \alpha \in S^{2},
\end{gathered}
$$

where $S^{2}$ is the unit sphere, $\alpha$ is a given unit vector. $A\left(\alpha^{\prime}, \alpha, k\right)$ is the scattering amplitude, and $q(x)$ is the potential. We assume that $q \in Q:=\{q: q=\bar{q}$ (real-valuedness), $q(x)=0$ for $\left.|x| \geq a, q \in L^{\infty}\right\}, k>0$ is fixed. The solution to (1.1)-(1.2) is called the scattering solution. We denote $B_{a}$ the ball centered at the origin with radius $a, u_{0}:=\exp (i k \alpha \cdot x), M_{k}:=\left\{z: z \in \mathbb{C}^{3}, z \cdot z=k^{2}\right\}$, $z \cdot y:=\sum_{j=1}^{3} z_{j} y_{j}, M_{1}:=M, M_{k}$ is a non-compact algebraic variety in $\mathbb{C}^{3}$, $M \cap \mathbb{R}^{3}=S^{2}$. 
The IPS consists of finding $q \in Q$, given $A\left(\alpha^{\prime}, \alpha\right):=A\left(\alpha^{\prime}, \alpha, k\right)$ for all $\alpha^{\prime}, \alpha \in$ $S^{2}$. We fix $k=1$ without loss of generality.

The general method for a study of inverse scattering problems, based on property $C$, was introduced in [4] and in [20] a detailed account of this method is given and its many applications are described. (See [4-42]). Later [29-31,38] the author introduced the notion of property $C$ with constraints and found several applications of this notion in inverse scattering.

Let us define property $C$ : let $L_{j}$ be linear partial differential expressions, $j=1,2, N_{j}=N_{j}(D):=\left\{w: L_{j} w=0\right.$ in $\left.D\right\} . D \subset \mathbb{R}^{n}, n \geq 2, D$ is a bounded domain; consider subsets of $N_{j}$ for which the products $w_{1} w_{2}$ are defined, $w_{j} \in N_{j}$; let $f \in L^{p}(D), p \geq 1$, and assume that the equation

$$
\int_{D} f(x) w_{1} w_{2} d x=0 \quad \forall w_{j} \in V_{j}
$$

implies $f(x)=0$. Then we say that the pair $\left\{L_{1}, L_{2}\right\}$ has property $C$.

This definition is rather flexible: we can take $f(x)$ not in $L^{p}(D)$ but in a different space, including a space of distributions.

The definition of property $C$ with constraints is similar, but $w_{j}$ in (1.3) runs not through all of $N_{j}$ but through a subset of $N_{j}$ of finite codimension.

For example, $w_{j}$ may satisfy finitely many linear constraints:

$$
\left(w_{j}, h_{m}\right)=0, \quad 1 \leq m \leq M_{j}<\infty,
$$

where $\left(w_{j}, h_{m}\right)$ is a linear functional on $N_{j}$. This functional can be defined by a function or distribution with support in $\bar{D}=D \cup \partial D$.

Let $l_{j}=\nabla^{2}+k^{2}-q_{j}(x), q_{j} \in Q$.

Theorem $1.1[29,30,38]$. The pair $\left\{l_{1}, l_{2}\right\}$ has property $C$ with constraints.

Let $A_{j}$ be the scattering amplitude corresponding to $q_{j}, j=1,2$. From Theorem 1.1 one derives:

Theorem 1.2 [32,33]. If $q \in Q, j=1,2$, and $A_{1}\left(\alpha^{\prime}, \alpha\right)-A_{2}\left(\alpha^{\prime}, \alpha\right)$ is a finite rank kernel in $L^{2}\left(S^{2}\right)$, then $q_{1}=q_{2}$.

Sketch of proof: Let $q_{1}-q_{2}:=p(x), A_{1}-A_{2}:=A$. Then one proves $[20, p .67]$ that

$$
-4 \pi A=\int p(x) u_{1}(x, \alpha) u_{2}\left(x,-\alpha^{\prime}\right) d x \quad \forall \alpha^{\prime}, \alpha \in S^{2} .
$$

If $A$ is a finite rank kernel, then $A=\sum_{m=1}^{M} a_{m}(\alpha) b_{m}\left(\alpha^{\prime}\right)$. Let $\left(\nu_{2}\left(\alpha^{\prime}\right), b_{m}\left(\alpha^{\prime}\right)\right)$ $=0,1 \leq m \leq M, \nu_{1}(\alpha)$ be arbitrary. Multiply $(*)$ by $\nu_{1}(\alpha) \nu_{2}\left(\alpha^{\prime}\right)$ and integrate over $\bar{S}^{2} \times S^{2}$ to get 


$$
\begin{gathered}
0=\int_{B_{\alpha}} p(x) w_{1}(x) w_{2}(x) d x . \\
w_{1}:=\int_{S^{2}} u_{1}(x, \alpha) \nu_{1}(\alpha) d \alpha, w_{2}:=\int_{S^{2}} u_{2}\left(x,-\alpha^{\prime}\right) \nu_{2}\left(\alpha^{\prime}\right) d \alpha^{\prime} .
\end{gathered}
$$

The set $\left\{w_{1}\right\}$ is dense in $N_{1}:=\left\{w: l_{1} w=0\right.$ in $\left.B_{a}\right\}$ in $L^{2}\left(B_{a}\right)$ norm when $\nu$ run through all of $L^{2}\left(S^{2}\right)$, while the set $\left\{w_{2}\right\}$ has codimension $\leq M$ in $N_{2}$ (see [33]). By Theorem 1.1, the set $\left\{w_{1} w_{2}\right\}$ is total in $L^{2}\left(B_{a}\right)$. Therefore $(* *)$ implies $p(x)=0$.

Note that the classical uniqueness theorem for ISP [20] says: if $A_{1}\left(\alpha^{\prime}, \alpha\right)=$ $A_{2}\left(\alpha^{\prime}, \alpha\right) \forall \alpha^{\prime}, \alpha \in S^{2}$ and $q_{j} \in Q$, then $q_{1}=q_{2}$. Thus, Theorem 2 is a far-reaching generalization of the classical uniqueness theorem. Results in $[29,30,38]$ cover also the case of $I O S$ for various boundary conditions, and contain results on inverse spectral problems and inverse boundary value problems. Roughly speaking, it is proved in [29.30,38] that one can drop any finite number of spectral data or boundary data. and uniqueness of the solutions to inverse spectral problems and inverse boundary value problems is still guaranteed.

Assume that $q=q(r)$, that is, the potential is spherically symmetric, and that all the phase shifts with $\ell>\ell_{0}$ vanish, where $\ell_{0}$ is an arbitrary large fixed integer. Then, as follows from Theorem $1.2, q(r)=0$.

The IGS problem consists of finding an inhomogeneity $v(x)$ in the velocity profile from the surface scattering data. The governing equation is

$$
\left[\nabla^{2}+k^{2}+k^{2} v(x)\right] U=-\delta(x-y) \text { in } \mathbb{R}^{3}, \quad k=\text { const }>0,
$$

$U$ satisfies the radiation condition, $v(x) \in Q$. supp $v \subset \mathbb{R}_{-}^{3}, \mathbb{R}_{-}^{3}:=\{x$ : $\left.x_{3}<0\right\}$. The surface scattering data are the values $U(x, y):=U(x, y, k) \forall x$, $\forall y \in P:=\left\{x: x_{3}=0\right\}, k>0$ is a fixed constant.

The IOS problem consists of finding an obstacle $D$ and the boundary condition on $\partial D:=S$ from the scattering amplitude $A\left(\alpha^{\prime}, \alpha\right), k>0$ is fixed. The scattering solution is defined by equation $(1.1)$ in $D^{\prime}:=\mathbb{R} \backslash D$ with $q(x)=0$, equation (1.2), and the boundary condition:

$$
u_{N}+\sigma(s) u=0 \text { on } S:=\partial D, \quad \sigma(s) \geq 0,
$$

where $u_{N}$ is the normal derivative, $N$ is the outer unit normal to $s, \sigma(s) \in$ $C(S)$. It is proved in [41] for Liapunov's boundaries and in [43] for Lipschitz's and even less smooth boundaries (see also [44]) that IOS has at most one solution. It is proved in $[4,20]$ that $I G S$ has at most one solution. We do not give the strongest results from [20] because of the lack of space. For example, in IPS and IOS the uniqueness results are proved in $[20,41]$ for the data known $\forall \alpha^{\prime} \in \tilde{S}_{1}^{2}$, and all $\alpha \in \tilde{S}_{2}^{2}$ where $\tilde{S}_{j}^{2}$ are arbitrary small open subsets of 
$S^{2}$, in $I G S$ the surface of data can be given on arbitrary small open subsets of $P$, the assumptions on $q(x)$ and $v(x)$ can be relaxed, etc.

In section 2 we describe an inversion algorithm for IPS and give an error estimate for this algorithm. In section 3 we formulate a variational principle equivalent to inverse scattering problems. In section 4 we outline a low-frequency data inversion in IGS. In section 5 an asymptotic theory for scattering by a small inhomogeneity is given. In section 6 two old open problems are stated.

\section{Inversion of Noisy Fixed-Energy Discrete Scattering Data}

Assume that $A_{\delta}\left(\alpha^{\prime}, \alpha\right)$ is known, $\sup _{\alpha^{\prime}, \alpha \in S^{2}}\left|A_{\delta}-A\right|<\delta$, so that $A_{\delta}\left(\alpha^{\prime}, \alpha\right)$ is the noisy scattering amplitude measured at a fixed energy $(k=1)$ with $\delta>0$ being the noise level. We do not assume any statistical properties of the noise. At the end, it will be clear that only the values of $A_{\delta}$ at a discrete set of $\alpha^{\prime}, \alpha \in S^{2}$ are used in the inversion algorithm. We fix an arbitrary $\xi \in \mathbb{R}^{3}$ and chose $\theta^{\prime}, \theta$ such that

$$
\theta^{\prime}-\theta=\xi, \quad|\theta| \rightarrow \infty, \quad \theta^{\prime}, \theta \in M .
$$

This can be done easily and explicitly: choose the coordinates in which $\xi=$ $t e_{3}, t>0, e_{j}$ are unit orthonormal basis vectors, $\theta^{\prime}=\frac{t}{2} e_{3}+y, \theta=-\frac{t}{2} e_{3}+y$, $y_{3}=0,|y| \rightarrow \infty, y_{1}^{2}+y_{2}^{2}=y \cdot y=1-\frac{t^{2}}{4}$. There are infinitely many $y$ that satisfy these requirements. Let

$$
\tilde{q}(\xi):=\int_{\mathbb{R}^{3}} \exp (-i \xi \cdot x) q(x) d x .
$$

Fix arbitrary $a_{1}$ and $b$ such that $a<a_{1}<b$. Let $N(\delta):=\left[\frac{|\ln \delta|}{\ln |\ln \delta|}\right]$, where $[x]$ is the closest to $x$ integer. Define

$$
A_{\delta \ell}(\alpha):=\left(A_{\delta}\left(\alpha^{\prime}, \alpha\right), Y_{\ell}\left(\alpha^{\prime}\right)\right)_{L_{2}\left(S^{2}\right)}
$$

where $Y_{\ell}(\alpha):=Y_{\ell m}(\alpha)$ are the orthonormal spherical harmonics. Let

$$
\hat{A}_{\delta}\left(\theta^{\prime}, \alpha\right):=\sum_{\ell=0}^{N(\delta)} A_{\delta \ell}(\alpha) Y_{\ell}\left(\theta^{\prime}\right)
$$

where the summation is taken over $\ell$ and also over $m,-\ell \leq m \leq \ell, Y_{\ell}(\theta)$ is naturally defined for all $\theta \in M$. Let $h_{\ell}(r)$ be the spherical Hankel function normalized so that $h_{\ell}(r) \sim \frac{\exp (i r)}{r}$ as $r \rightarrow \infty$, and let

$$
\hat{u}_{\delta}(x, \alpha):=\exp (i \alpha \cdot x)+\sum_{\ell=0}^{N(\delta)} A_{\delta \ell}(\alpha) Y_{\ell}\left(\alpha^{\prime}\right) h_{\ell}(r) .
$$


Define

$$
\rho_{\delta}(\nu):=\exp (-i \theta \cdot x) \int_{S^{2}} u_{\delta}(x, \alpha) \nu(\alpha) d \alpha-1, \quad \theta \in M, \quad \nu \in L^{2}\left(S^{2}\right),
$$

$\mu(\delta)=\exp [-\gamma N(\delta)], \gamma:=\ln \frac{a_{1}}{a}>0,\|\rho\|:=\|\rho\|_{L^{2}\left(B_{b} \backslash B_{a_{1}}\right)}$. Let $c>0$ be a sufficiently large constant, $\xi \in \mathbb{R}$, and $\theta^{\prime}, \theta$ satisfy $(2.1)$. Consider the variational problem:

$$
\begin{gathered}
|\theta|=\sup , \quad \sup |\theta|:=\vartheta(\delta) \\
\left\|\rho_{\delta}(\nu)\right\|+\|\nu\|_{L^{2}\left(S^{2}\right)} \exp (|\operatorname{Im} \theta| b) \mu(\delta) \leq c|\theta|^{-1}
\end{gathered}
$$

Lemma $2.1 \vartheta(\delta) \geq c_{0} \frac{|\ln \delta|}{(\ln |\ln \delta|)^{2}}, c_{0}=$ const $>0$.

Let $\theta(\delta)$, and $\nu_{\delta}(\alpha)$ be any approximate solution to (2.2) in the following sense: (2.1) and (2.3) hold and $|\theta(\delta)| \geq \frac{1}{2} \vartheta(\delta)$. Define

$$
\hat{q}:=-4 \pi \int_{S^{2}} \hat{A}_{\delta}\left(\theta^{\prime}(\delta), \alpha\right) \nu_{\delta}(\alpha, \theta(\delta)) d \alpha .
$$

Theorem 2.1 One has

$$
|\hat{q}-\tilde{q}(\xi)| \leq c_{1} \frac{(\ln |\ln \delta|)^{2}}{|\ln \delta|}
$$

where $c_{1}$ can be chosen uniformly for $\xi \in \mathbb{R}^{3}$ and $q \in \boldsymbol{N} \subset Q$, where $\boldsymbol{k}$ : is an arbitrary fixed compact subset of $Q$.

Formula (2.4) gives an inversion algorithm and (2.5) is its error estimate. Since calculation of (2.4) by a cubature formula requires the knowledge of $\hat{A}_{\delta}\left(\alpha^{\prime}, \alpha\right)$ at a discrete set only, formula (2.4) gives inversion procedure for noisy, discrete, fixed-energy, 3-D scattering data.

\section{Variational Principles Equivalent to Inverse Scattering Problems}

We give here only the variational principle equivalent to IPS and refer the reader to $[21,42]$ where similar principles for $I G S$ and $I O S$ are given.

The basic idea is to use not only the data fitting, but also the basic equation for the scattering solution, namely,

$$
u=u_{0}-\int_{\mathbb{R}^{3}} g q u d z, \quad g:=\frac{\exp (i|x-y|)}{4 \pi|x-y|}, \quad k=1 .
$$

Consider the variational principle:

$$
\begin{gathered}
\mathcal{F}(p, f):=\left\|-4 \pi A\left(\alpha^{\prime}, \alpha\right)-\int_{B_{a}} \exp \left(-i \alpha^{\prime} \cdot y\right) f(y, \alpha) d y\right\|_{L^{2}\left(S^{2} \times S^{2}\right)} \\
+\left\|f(x, \alpha)-p(x) u_{0}(x, \alpha)+p(x) \int_{B_{\alpha}} g(x, z) f(z, \alpha) d z\right\|_{L^{2}\left(B_{a} \times S^{2}\right)} \\
=\min
\end{gathered}
$$


Theorem 3.1 If $A\left(\alpha^{\prime} . \alpha\right)$ is the scattering amplitude corresponding to a potential $q \in Q$, then $\min \mathcal{F}(p, f)=0$, where min is taken over $p \in L^{2}\left(B_{q}\right)$ and $f(x, \alpha) \in L^{2}\left(B_{a} \times S^{2}\right) ; \min \mathcal{F}(p, f)$ is attained at the pair $\{q, q u(x, \alpha)\}$ and only at this pair. Here $q \in Q$ is the unique potential corresponding to $A\left(\alpha^{\prime}, \alpha\right)$ and $u(x, \alpha)$ is the corresponding scattering solution.

Remark 3.1 Unfortunately we cannot give any stability results for the solution to (3.2) with noisy data $A_{\delta}\left(\alpha^{\prime}, a\right)$ in place of the exact data $A\left(\alpha^{\prime}, \alpha\right)$ in (3.2).

\section{Inversion of Low-Frequency Surface Scattering Data}

Consider (1.4) and assume that the data $U(x, y, k), \forall x, y \in P, \forall k \in\left(0, k_{0}\right)$ are known, where $k_{0}>0$ is an arbitrary small number. The usual integral equation equivalent to (1.4) (with the radiation condition) is:

$$
U=g+k^{2} \int_{B_{a}} g(x, y, k) v(z) U(z, y, k) d z, \quad g:=\frac{\exp (i k|k-y|)}{4 \pi|x-y|} .
$$

It is proved in [41] that (4.1) is uniquely solvable for sufficiently small $k$ and the following limit exists:

$$
f(x, y):=16 \pi^{2} \lim _{k \rightarrow 0} \frac{U-g}{k^{2}}=\int_{B_{a}} \frac{v(x) d z}{|x-z||z-y|}, \quad x, y \in P .
$$

The function $f(x, y)$ is known if $U(x, y)$ is known $\forall x, y \in P$. Equation (4.2) is first kind Fredholm equation for $v(z)$. It is proved in [41] that this equation has at most one solution, this solution is found analytically, and necessary and sufficient condition for a function $f(x, y)$ to be representable by the righthand side of (4.2) are given [20,21,41]. Numerical experiments in solving (4.2) are reported in [20]. The problem is highly ill-posed, but it was possible to identify numerically the support of $v(x)$ from noisy discrete surface data. A number of other geophysical inverse problems were studied by the above methodology (well-to-well exploration, induction logging. etc, see $[20,21,41]$ ). However, in many cases it is desirable to get less information about the inhomogeneity $v(x)$, but to get it in a computationally stable and easy (nonintensive computationally) way. Such a way is discussed in the next section.

\section{$5 \quad$ Asymptotic Inverse Scattering Theory for Small Inhomogeneities}

Consider IGS problem and assume that 


$$
\begin{gathered}
\text { supp } v:=D, \quad \operatorname{diam}(D)=d, \\
c_{1} d^{3} \leq|D|:=\text { meas } D \leq c_{2} d^{3}, \\
\frac{\int_{D}|v| d x}{\left|\int_{D} v d x\right|} \leq c, \quad \sup |v| \leq c .
\end{gathered}
$$

Given $U(x, y, k)$ for $x$ and $y$ running through some subsets of $P$ and for $k$ fixed or $k$ running through some finite number of wave numbers, we want to find the location of $D$ and get some idea about its geometry and the intensity $v_{D}:=\int_{D} v d x$.

In [1-3] more general assumptions are made: $\nabla^{2}$ is replaced by a second-order elliptic operator. Let $(U-g) k^{-2}:=f(x, y)$. We suppressed $k$-dependence in what follows. Equation (4.1) implies

$$
f(x, y) \approx g(x, \tilde{z}) g(\tilde{z}, y) v_{D}, \quad v_{D}:=\int_{D} v d x
$$

where $\tilde{z}$ is a point in a neighborhood of $D$.

Let $\xi_{i}=\left(x_{i}, y_{i}\right)$ be a data point, $\hat{g}\left(\xi_{i}, z\right):=g\left(x_{i}, z\right) g\left(z, y_{i}\right), i \in I$, where $I$ is a finite set of indices. By $(5.2), f(\xi)$ is approximately proportional to $\hat{g}(\xi, \tilde{z})$ for some $\tilde{z}$. Let us estimate $\tilde{z}$ by finding global maximum of the correlation coefficient

$$
r(z):=\frac{\left|\sum f\left(\xi_{i}\right) \hat{g}\left(\xi_{i}, z\right)\right|^{2}}{\sum\left|f\left(\xi_{i}\right)\right|^{2} \sum\left|\hat{g}\left(\xi_{i}, z\right)\right|^{2}}=\max
$$

where the summation is over $i \in I$. Define

$$
z_{0}:=\int_{D} z v(z) d z / v_{D}
$$

One can prove [1] the following

\section{Theorem 5.1 Global minimizer $\tilde{z}$ of (5.3) satisfies the following equation.

$$
\tilde{z}=z_{0}+C\left(d^{\frac{3}{2}}\right) \text { as } d \rightarrow 0
$$

A similar result holds when the exact data $U\left(\xi_{i}\right)$ are replaced by the noisy data $U\left(\xi_{i}\right)+n_{i}$, where $n_{i}$ are independent random variables with zero mean value and variance $\sigma^{2}$ (see [1]).

If $v$ does not change sign then $\tilde{z}$ belongs to the convex hull of $D$. If, in addition, $D$ is convex, then $z \in D$. If $D$ is centrally symmetric and connected and $v(x)=v(-x)$ in the coordinate system with the origin at the center of symmetry, then $\tilde{z}=C\left(d^{\frac{3}{2}}\right)$.

We can now describe a very simple practical algorithm for localization of the small inhomogeneity $v(x)$ : calculate $r(z)$ by formula (5.3) and find its global minimizer $\tilde{z}$. The point $\tilde{z}$ determines the position of $D$ with the error $C\left(d^{\frac{3}{2}}\right)$ as $d \rightarrow 0$. If $\tilde{z}$ is found then an estimate of $v_{D}$ is calculated by the formula

$$
\tilde{v}_{D}:=\frac{\operatorname{Re} \sum f\left(\xi_{i}\right) \hat{g}\left(\xi_{i}, \tilde{z}\right)}{\sum\left|\hat{g}\left(\xi_{i}, \tilde{z}\right)\right|^{2}}
$$

which follows from (5.2). One has 


\section{Theorem 5.2.}

$$
\tilde{v}_{d}=v_{d}\left[1+C\left(d^{\frac{3}{2}}\right)\right] \text { as } d \rightarrow 0
$$

One can get additional, useful practically, information about $v(z)$ from the surface scattering date, in particular, one can estinate the first and second moments of $v(z)$. (See [1-2] for details.)

A similar approach can be used in IPS ([3]). Assuming that

$$
d^{2}\left(k^{2}+\sup |q(x)|\right) \ll 1 \text { and } d \sup |q| \ll k,
$$

where $d=\operatorname{diam}(\operatorname{supp} q)$, supp $q:=D \subset \mathbb{R}^{3}$, one can give a computationally easy and stable method for locating $D$, finding $q_{D}:=\int_{D} q(x) d x$, the intensity of the potential, and its first and second moments.

Let us sketch the method. We have the well-known equations

$$
\begin{gathered}
A\left(\alpha^{\prime}, \alpha\right)=-\frac{1}{4 \pi} \int_{D} \exp \left(-i k \alpha^{\prime} \cdot x\right) q(x) u(x, \alpha) d x \\
U(x, \alpha)=\exp (i k \alpha \cdot x)-\int_{D} g(x, y, k) q(y) u(y, \alpha) d y, \quad \alpha, \alpha^{\prime} \in S^{2} .
\end{gathered}
$$

Thus

$$
\begin{gathered}
-4 \pi A\left(\alpha^{\prime}, \alpha\right)= \\
\int_{D} q(z) \exp \left[i k\left(\alpha-\alpha^{\prime}\right) \cdot x\right] d x-\int_{D} d x \exp (-i k \alpha \cdot x) q(x) \int_{D} g(x, y, k) q u d y
\end{gathered}
$$

Let

$$
x_{0}:=\int_{D} x q(x) d x / q_{D}, \quad \kappa:=k^{2}+Q, \quad Q:=\sup _{x \in \mathbb{R}^{3}}|q| .
$$

One can prove that (5.9) implies

$$
-4 \pi A\left(\alpha^{\prime}, \alpha\right)=\exp \left[i k\left(\alpha-\alpha^{\prime}\right) \cdot x_{0}\right] q_{D}\left[1+C\left(\kappa d^{2}\right)\right], \quad d \rightarrow 0 .
$$

Thus

$$
\begin{gathered}
4 \pi\left|A\left(\alpha^{\prime}, \alpha\right)\right|=\left|q_{D}\right|\left(1+\kappa d^{2}\right) ; \quad 0 \leq \arg \left[-A\left(\alpha^{\prime}, \alpha\right)\right]:=c\left(\alpha^{\prime}, \alpha\right)<2 \pi \\
c\left(\alpha^{\prime}, \alpha\right)=k\left(\alpha-\alpha^{\prime}\right) \cdot x_{0}+2 \pi n\left(\alpha^{\prime}, \alpha\right)+C\left(\kappa d^{2}\right)
\end{gathered}
$$

where $n\left(\alpha^{\prime}, \alpha\right)$ is an integer.

Let $\beta:=\alpha-\alpha^{\prime}$. If $\alpha, \alpha^{\prime} \in S^{2}$ then $|\beta| \leq 2$. If $\beta$ is given, $\beta \in \mathbb{R}^{3},|\beta| \leq 2$, and $\mathcal{P}$, a plane containing the origin, is fixed, then one can find uniquely $\alpha$, $\alpha^{\prime} \in S^{2} \cap \mathcal{P}$, such that $\beta=\alpha^{\prime}-\alpha$. Therefore (5.13) can be written as

$$
c(\beta, P)=k \beta \cdot x_{0}+2 \pi n(\beta, \mathcal{P})+C\left(\kappa d^{2}\right), \quad|\beta| \leq 2 .
$$

From the second equation $(5.12)$ it follows that $c(\beta . \mathcal{P})$ is discontinuous only for those $\beta$ for which $c(\beta, \mathcal{P})=0$ or $c(\beta, \mathcal{P})=2 \pi-0$. Suppose the origin is inside $B_{a} \supset D:=\operatorname{supp} q(x)$ and assume that $x_{0} \in B_{a}$. This will happen, for example, if the ratio $\frac{\int_{B_{a}}|q| d x}{\left|\int_{B_{a}} q d x\right|}$ is not too large. For instance, if $q(x)$ does not change sign, then this ratio is 1 and $x_{0} \in B_{a}$. Define $\triangle:=\pi(k a)^{-1}$ and let $\left|\beta_{2}-\beta_{1}\right|<\triangle$. Then $\left|k\left(\beta_{2}-\beta_{1}\right) \cdot x_{0}\right| \leq k a\left|\beta_{2}-\beta_{1}\right|<\pi$. Fix any $\beta_{1} \neq \beta_{2}$ such that $\left|\beta_{1}-\beta_{2}\right|<\triangle$. Consider the following three possibilities: 
- 1) $-\pi+C\left(\kappa d^{2}\right)<c_{2}-c_{1}<\pi-C\left(\kappa d^{2}\right)$, where $c_{j}=c\left(\beta_{j}, \mathcal{P}_{j}\right)$; then $\left(\beta_{2}-\beta_{1}\right) \cdot x_{0}=k^{-1}\left(c_{2}-c_{1}\right)+C\left(\kappa k^{-1} d^{2}\right)$;

- 2) $c_{2}-c_{1}>\pi+C\left(\kappa d^{2}\right)$, then $n_{2}=n_{1}+1, n_{j}:=\left(\beta_{j}, \mathcal{P}_{j}\right)$; thus $\left(\beta_{2}-\beta_{1}\right) x_{0}=$ $k^{-1}\left[c_{2}-c_{1}-2 \pi+C\left(\kappa d^{2}\right)\right]$

- 3) $c_{2}-c_{1}<-\pi-C\left(\kappa d^{2}\right)$, then $n_{2}-n_{1}=-1$, and $\left(\beta_{2}-\beta_{1}\right) x_{0}=$ $k^{-1}\left[c_{1}-c_{1}+2 \pi+C\left(\kappa d^{2}\right)\right]$.

Let us estimate the point $x_{0}$ which localizes the support of the potential.

Let $\left\{\beta_{j}, c_{j}\right\}$ be the data. For all pairs $j_{1}, j_{2}$ such that $\left|\beta_{j_{1}}-\beta_{j_{2}}\right|<\Delta, \beta_{j_{1}} \neq$ $\beta_{j_{2}}$, consider the equations

$$
x \cdot b_{l}=k^{-1} p_{l}, \quad 1 \leq l \leq L, \quad b_{l}:=\beta_{j_{2}}-\beta_{j_{1}}, \quad p_{l}:=c_{j_{2}}-c_{j_{1}}+2 \pi s .
$$

Index $l$ numerates the pairs $\left\{j_{1}, j_{2}\right\}, s=0,1$, or -1 according to which of the possibilities 1), 2) or 3) occurs.

Solve (5.15) for $x$ by solving the system

$$
B^{t} B \tilde{x}=k^{-1} B^{t} p, \quad p=\left(\begin{array}{c}
p_{1} \\
\vdots \\
p_{l}
\end{array}\right), \quad B=\left(\begin{array}{ccc}
b_{11} & b_{12} & b_{13} \\
\vdots & & \\
b_{L 1} & b_{L 2} & b_{L 3}
\end{array}\right)
$$

If the vectors $\frac{\beta}{|\beta|}, \beta:=\alpha^{\prime}, \alpha$, are distributed uniformly on $S^{2}$ and if there are many pairs $\left\{\beta_{j_{1}}, \beta_{j_{2}}\right\}, \beta_{j_{1}} \neq \beta_{j_{2}}$, such that $\left|\beta_{j_{1}}-\beta_{j_{2}}\right|<\Delta$, then one can find $\tilde{x}$ from $(5 . .16)$.

Theorem 5.3 One has $\left|\tilde{x}-x_{0}\right|=C\left(k d^{2}+k^{-1} Q d^{2}\right)$ as $d \rightarrow 0$.

If $\tilde{x}$ is found, then one estimates the intensity of the potential, $q_{D}$, by the formula

$$
\tilde{q}_{D}=q_{D}\left[1+C\left(d^{2} k^{2}+Q d^{2}\right)\right] \text { as } d \rightarrow 0 .
$$

As above, one can find the first and second moments of $q(x)$ from the scattering data.

\section{Open Problems}

We mention just two major problems each of which has been open for about half a century:

- 1) Do the data $A\left(\alpha^{\prime}, \alpha_{0}, k\right)$ (or $A(-\alpha, \alpha, k)$ ) determine $q(x)$ uniquely? Here $\alpha_{0} \in S^{2}$ is fixed, $\alpha^{\prime}, \alpha$ run through all of $S^{2}$ and $k$ runs through $(0, \infty)$

- 2) Do the data $A\left(\alpha^{\prime}, \alpha_{0}, k_{0}\right)$ determine $D$ uniquely?

Here $\alpha_{0} \in S^{2}$ and $k_{0}>0$ are fixed, $\alpha^{\prime}$ runs through an open subset of $S^{2}$, and $D \subset \mathbb{R}^{3}$ is a bounded domain with a Lipschitz (or smooth) boundary on which the Dirichlet (or Neumann, or Robin) boundary condition is assumed. 


\section{References}

[1] KATSEVICH A.I. \& RAMM A.G., Approximate inverse geophysical scattering on a small body, SIAM J. Appl. Math., 56, N1, (1996), 192-218.

[2] KATSEVICH A.I. \& RAMM A.G., Inverse geophysical and potential scattering on a small body, in the book: Experimental and Numerical Methods for Solving IIl-Posed Inverse Problems: Medical and Nonmedical Applications, vol. SPIE2570, (1995), 151-162.

[3] KATSEVICH A.I. \& RAMM A.G., Approximate solution to inverse scattering problem for potentials with small support, Math. Meth. in the Appl. Sci.,19, N14, (1996), 1121-1134.

[4] RAMM A.G. Completeness of the products of solutions to PDE and uniqueness theorems in inverse scattering, Inverse problems, 3, (1987), Li7-L82

[5] RAMM A.G., A uniqueness theorem for two-parameter inversion. Inverse Probl., 4, (1988), L7-10.

[6] RAMM A.G., A uniqueness theorem for a boundary inverse problem, Inverse Probl., 4, (1988), L1-5.

[7] RAMM A.G., Recovery of the potential from fixed energy scattering data. Inverse Problems, 4, (1988), 877-886; 5, (1989) 255.

[8] RAMM A.G., A simple proof of uniqueness theorem in impedance tomography. Appl. Math. Lett., 1, N3, (1988), 287-290.

[9] RAMM A.G., Uniqueness theorems for multidimensional inverse problems with unbounded coefficients. J. Math. Anal. Appl, 134, (1988), 211-253; 139, (1989), 302; 136, (1988), 568-574.

[10] RAMM A.G., Numerical method for solving 3D inverse problems of geophysics, J. Math. Anal. Appl., 136, (1988), 352-356.

[11] RAMM A.G., Multidimensional inverse problems: Uniqueness theorems, Appl. Math. Lett., 1, N4, (1988), 377-380.

[12] RAMM A.G., Multidimensional inverse scattering problems and completeness of the products of solutions to homogeneous PDE. Zeitschr. f. angew. Math. u. Mech., 69, (1989) N4, T13-T22.

[13] RAMM A.G., Numerical method for solving 3D inverse problems with complete and incomplete data, In the book: "Wave Phenomena", Springer-Verlag, New York 1989, (eds. L. Lam and H. Morris), 34- 43.

[14] RAMM A.G., Numerical recovery of the 3D potential from fixed energy incomplete scattering data, Appl. Math. Lett. 2, N1, (1989), 101-104.

[15] RAMM A.G., Property $\mathrm{C}$ and uniqueness theorems for multidimensional inverse spectral problem, Appl. Math. Lett.. 3, (1990). 57-60.

[16] RAMM A.G., Stability of the numerical method for solving the $3 D$ inverse scattering problem with fixed energy data, Inverse problems 6, (1990), L7-12.

[17] RAMM A.G., Completeness of the products of solutions of PDE and inverse problems, Inverse Probl., 6, (1990), 643-664.

[18] RAMM A.G., Uniqueness result for inverse problem of geophysics I, Inverse Probl., 6, (1990), 635-642.

[19] RAMM A.G., Uniqueness theorems for geophysical problems with incomplete surface data. Appl. Math. Lett., 3, (1990), N4, 41-44.

[20] RAMM A.G., Multidimensional inverse scattering problems, LONGMAN/WILEY, New York, 1992, pp. 1-386. 
[21] RAMM A.G., Multidimensional inverse scattering problems, Mir Publishers, Moscow, 1994 (Russian), pp. 1-496.

[22] RAMM A.G., Stability of the numerical method for solving 3D inverse scattering problem with fixed energy data, J.f.dic reine und angew. Math. 414, (1991), 1-21.

[23] RAMM A.G., Stability of the inversion of 3D fixed-frequency data, J. Math. Anal. Appl, 169, N2(1992), 329-349.

[24] RAMM A.G., Stability of the solution to 3D fixed-energy inverse scattering problem, J. Math. Anal. Appl., 170, N1 (1992), 1-15.

[25] RAMM A.G., Finding conductivity from boundary measurements, Comp. $\&$ Math. with Appl, 21, N8, (1991), 85-91

[26] RAMM A.G., Stability estimates in inverse scattering, Acta Appl. Math., 28, N1, (1992), 1-42.

[27] RAMM A.G., Numerical solution of 3D inverse scattering problems with noisy discrete fixed-energy data, Appl. Math. Lett., 5, N6, (1992), 15-18.

[28] RAMM A.G., Property C and inverse problems, ICM-90 Satellite Conference Proceedings, Inverse Problems in Engineering Sciences, Proc. of a conference held in Osaka, Japan, Aug. 1990, Springer Verlag, New York, 1991, pp. 139-144.

[29] RAMM A.G., Property $C$ with constraints and inverse spectral problems with incomplete data, J. Math. Anal. Appl, 180, N1, (1993), 239-244

[30] RAMM A.G., Property C with constraints and inverse problems, J. of Inverse and Ill-Posed Problems, 1, N3 (1993), 227-230.

[31] RAMM A.G., Property C and applications.Math. Comp. Modelling, 18, N1, (1993), 1-4.

[32] RAMM A.G., Scattering amplitude is not a finite rank kernel in the basis of spherical harmonics, Appl. Math. Lett,, 6, N5, (1993), 89-92.

[33] RAMM A.G., Scattering amplitude is not a finite-rank kernel, J. of Inverse and Ill-Posed Problems, 1, N4, (1993), 349-354.(with P.Stefanov)

[34] RAMM A.G., Scattering amplitude as a function of the obstacle, Appl. Math. Lett., 6, N5, (1993), 85-87.

[35] RAMM A.G., Multidimensional inverse scattering: solved and unsolved problems, Proc. Intern. Confer. on Dynamical Syst.and Applic., Vol.1, Atlanta, (1994), pp.287-296.(Eds. G.Ladde and M.Sambandham)

[36] RAMM A.G., Stability of the solution to inverse obstacle scattering problem, J. Inverse and Ill-Posed Problems, 2, N3, (1994), 269-275.

[37] RAMM A.G., Stability estimates for obstacle scattering, J.Math.Anal.Appl., 188, N3, (1994), 743-751.

[38] RAMM A.G., Property C with constraints, Compt. Rendus, Paris, ser 1, 321, N 11, (1995), 1413-1417.

[39] RAMM A.G., A formula for inversion of boundary data, J. of Inverse and Ill-Posed Problems, 3, N5, (1995), 411-415.

[40] RAMM A.G., Finding potential from the fixed-energy scattering data via D-N map, J. of Inverse and Ill-Posed Problems, 4, N2,(1996), 145-152.

[41] RAMM A.G., Scattering by obstacles, D. REIDEL, Dordrecht, 1986.

[42] RAMM A.G., Numerical method for solving inverse scattering problems, Doklady of Russian Acad. of Sci., 337, N1, (1994), 20-22

[43] RAMM A.G., Uniqueness theorems for inverse obstacle scattering problems in Lipschitz domains, Applic. Analysis, 59, (1995), 377-383. 
[44] RAMM A.G., RUIZ A. Existence and uniqueness of scattering solutions in non-smooth domains, J. Math. Anal. Appl, 201 , (1996), 329-338. 